\title{
Visit-to-visit variability of lipid and cardiovascular events in patients with familial hypercholesterolemia
}

\author{
Ye-Xuan Cao ${ }^{1,2 \#}$, Liang $\mathrm{Li}^{3 \#}$, Hui-Wen Zhang ${ }^{1}$, Jing-Lu Jin ${ }^{1}$, Hui-Hui Liu ${ }^{1}$, Yuan-Lin Guo ${ }^{1}, \mathrm{Na}-\mathrm{Qiong} \mathrm{Wu}^{1}$, \\ Cheng-Gang Zhu ${ }^{1}$, Qian Dong ${ }^{1}$, Rui-Xia Xü ${ }^{1}$, Jing Sun ${ }^{1}$, Jian-Jun Li $^{1} \wedge$ \\ ${ }^{1}$ State Key Laboratory of Cardiovascular Disease, Fuwai Hospital, National Center for Cardiovascular Diseases, Chinese Academy of Medical \\ Sciences, Peking Union Medical College, Beijing, China; ${ }^{2}$ Department of Cardiology, Beijing Chaoyang Hospital Affiliated to Capital University \\ of Medical Science, Beijing, China; ${ }^{3}$ Department of Surgical Intensive Care Unit, State Key Laboratory of Cardiovascular Disease, Fuwai Hospital, \\ National Center For Cardiovascular Diseases, Chinese Academy of Medical Sciences, Peking Union Medical College Beijing, Beijing, China \\ Contributions: (I) Conception and design: JJ Li; (II) Administrative support: JJ Li, YL Guo; (III) Provision of study materials or patients: YL Guo, \\ NQ Wu; (IV) Collection and assembly of data: YX Cao, JL Jin, HW Zhang, HH Liu; (V) Data analysis and interpretation: YX Cao; (VI) Manuscript \\ writing: All authors; (VII) Final approval of manuscript: All authors. \\ "These authors contributed equally to this work. \\ Correspondence to: Prof. Jian-Jun Li, MD, PhD, FESC, FACC. State Key Laboratory of Cardiovascular Disease, Fuwai Hospital, National Center \\ for Cardiovascular Diseases, Chinese Academy of Medical Sciences, Peking Union Medical College, Beilishi Road 167, Beijing 100037, China. \\ Email: lijianjun938@126.com.
}

Background: Visit-to-visit variability in lipid has been suggested as a predictor of major adverse cardiovascular events (MACEs). However, no evidence exists on the prognostic value of lipid variability in patients with familial hypercholesterolemia (FH). This prospective cohort study aimed to investigate whether lipid variability affects future MACEs in patients with $\mathrm{FH}$ receiving standard lipid-lowering therapy.

Methods: A total of 254 patients with FH were consecutively enrolled and followed for MACEs. Variability in the triglyceride, total cholesterol, high-density lipoprotein-cholesterol (HDL-C), low-density lipoproteincholesterol (LDL-C) and lipoprotein (a) $[\mathrm{Lp}(\mathrm{a})]$ were evaluated from 3 months after discharge using the standard deviation (SD), coefficient of variation (CV) and variability independent of the mean (VIM).

Results: During a mean follow-up of 49 months, 22 (8.7\%) events occurred. Visit-to-visit variability in $\mathrm{Lp}$ (a) was significantly higher in the MACE group compared to the non-MACE group. In the multivariate Cox analysis, only Lp(a)-related parameters were independent predictors for MACEs. The hazard ratios and 95\% confidence intervals of each 1-SD increase of SD, CV, and VIM of Lp(a) were 1.42 (1.12-1.80), 1.50 (1.11-2.02) and 1.60 (1.16-2.22), respectively. Kaplan-Meier analysis revealed that patients with higher Lp(a) variability presented lower event-free survival. The results were consistent in various subgroups.

Conclusions: Our study firstly suggested that $\mathrm{Lp}$ (a) variability was associated with MACEs in real-world patients with $\mathrm{FH}$, which emphasized the importance of regular lipid monitoring in the patients with high risk.

Keywords: Lipid variability; familial hypercholesterolemia (FH); major adverse cardiovascular events (MACEs); lipoprotein (a) [Lp(a)]; low-density lipoprotein-cholesterol (LDL-C)

Submitted Jul 15, 2020. Accepted for publication Nov 09, 2020.

doi: 10.21037/atm-20-5324

View this article at: http://dx.doi.org/10.21037/atm-20-5324

$\wedge$ ORCID: 0000-0003-2536-4364. 


\section{Introduction}

Dyslipidemia has been widely considered as a primary cause of coronary artery disease (CAD) and this notion is supported by major clinical guidelines $(1,2)$. Elevated plasma low-density lipoprotein cholesterol (LDL-C) concentration revealed a dose-dependent effect on the development and progression of atherosclerotic plaque (3). A number of epidemiologic and genetic studies showed a causal relationship between LDL-C and major adverse cardiovascular events (MACEs) $(4,5)$. Lipoprotein (a) $[\mathrm{Lp}(\mathrm{a})]$, a mainly genetically determined lipoprotein particle, has recently been regarded as an independent risk factor for $\mathrm{CAD}$ and future cardiovascular outcomes (6). Familial hypercholesterolemia (FH), an autosomal dominant disorder, is characterized by lifelong raised LDL-C levels and increased risk of premature CAD $(7,8)$. Recent evidences have showed that $\mathrm{Lp}(\mathrm{a})$ level is significantly higher in the one third of $\mathrm{FH}$ patients and may play a pivotal role in CAD independent of LDL-C and other traditional risk factors (9-11). Our previous studies also showed that elevated $\mathrm{Lp}(\mathrm{a})$ at baseline and follow-up period was significantly associated with MACEs in FH patients (12).

Recently, emerging studies have demonstrated that visitto-visit intra-individual variability in various traditional risk factors may be potential novel prognostic markers for clinical outcomes in addition to mean levels (13). For example, increased visit-to-visit variability in blood pressure (BP) and lower variability in heart rate were associated with various adverse outcomes such as impaired cognition, stroke, or all-cause mortality $(14,15)$. Although major guidelines for management of dyslipidemia mainly focus on underlying the mean lipid concentration, the fluctuation in lipid has also attracted increasing concern in recent years. Several retrospective studies and post-hoc analysis of randomized controlled trials have reported a positive association between higher visit-to-visit variability in LDL-C and worse cardiovascular outcomes in the general population and patients with CAD (16-18). However, whether these findings could be extended to a broader lipoprotein profile such as $\mathrm{Lp}(\mathrm{a})$ has yet to be investigated. Moreover, no study has evaluated the role of lipid variability as a potential prognostic factor for future MACEs in patients with $\mathrm{FH}$.

Therefore, the aim of this prospective study was to investigate the prognostic significance of increased visit-tovisit variabilities in triglyceride (TG), total cholesterol (TC), high-density lipoprotein cholesterol (HDL-C), LDL-C and $\mathrm{Lp}(\mathrm{a})$ on MACEs in patients with $\mathrm{FH}$ receiving standard lipid-lowering therapy.

We present the study in accordance with the STROBE reporting checklist (available at http://dx.doi.org/10.21037/ atm-20-5324).

\section{Methods}

\section{Study design and population}

A total of 10,012 consecutive patients with dyslipidemia, who were hospitalized in Fuwai Hospital between March 2011 to November 2018 were enrolled. Among these patients, adult $\mathrm{FH}$ patients ( $\geq 18$ years old) were selected for the present study. Clinical FH diagnosis was made by Dutch Lipid Clinic Network (DLCN) criteria and only patients with DLCN scores $>6$ (definite and probable) were included (8). Untreated LDL-C levels were calculated by a correction factor depending on the type and potency of lipid-lowering drugs if patients received lipid-lowering drugs prior to admission (19). Patients were screened for genetic mutations in LDL receptor $(L D L R)$, Apolipoprotein $\mathrm{B}(A P O B)$, and proprotein convertase subtilisin/kexin type 9 (PCSK9) genes as previously reported $(7,8)$. The exclusion criteria included the following items: missed detailed data, homozygote $\mathrm{FH}$, had a history of heart failure (left ventricular ejection fraction $<45 \%$ ), severe liver and/or renal insufficiency, thyroid dysfunction, malignant disease, or with extreme high plasma TG $(\geq 500 \mathrm{mg} / \mathrm{dL})$. Considering PSCK9 inhibitor or lipoprotein apheresis have a drastic fluctuation on lipid levels and may exhibit a protective effect which might confusing the results, these patients were also excluded. All FH patients received standard statin therapy which was defined as rosuvastatin dose $20 \mathrm{mg}$ plus ezetimibe $10 \mathrm{mg}$ per day.

The study protocol was approved by the ethical review board of the Fuwai Hospital \& National Center for Cardiovascular Diseases, Beijing, China (approval ID: 2013442) and conducted in line with the Declaration of Helsinki (as revised in 2013). All patients enrolled in the study provided written informed consent.

\section{Baseline data collection}

Clinical data of each individual, including age, height, weight, BP, medical history, and family history were collected by experienced physicians and nurses at the time of enrollment. Hypertension was diagnosed if systolic BP $>140 \mathrm{mmHg}$ and/or diastolic $\mathrm{BP}>90 \mathrm{mmHg}$ on 3 
measurements on different days or using antihypertensive drugs. Diabetes was defined as a previous diagnosis of diabetes, fasting blood glucose $>7.0 \mathrm{mmol} / \mathrm{L}$, the 2 -h serum glucose of the oral glucose tolerance test $\geq 11.1 \mathrm{mmol} / \mathrm{L}$, glycated hemoglobin $(\mathrm{HbA} 1 \mathrm{c}) \geq 6.5 \%$ or treated with antidiabetic medication or insulin. Body mass index (BMI) was calculated as the weight (in kilograms) divided by the height (in meters) squared.

Blood samples were collected into EDTA-containing tubes after at least 12 -h fasting in the morning. The concentrations of plasma TC, TG, LDL-C, HDL-C, apolipoprotein A-I (apoAI) and apoB were measured using an automatic biochemistry analyzer (Hitachi 7150, Japan), while Lp(a) levels were assayed by an immunoturbidimetry method [LASAY Lp(a) auto; SHIMA laboratories] as previously described $(6,12)$. Plasma highsensitivity $\mathrm{C}$ reactive protein (hsCRP) levels were assayed by immunoturbidimetry (Beckmann Assay360, Bera, California). Other related biochemical and hematological indicators were measured according to standard tests. The intra- and inter-assay coefficients of variation for these assays were all $<8 \%$.

\section{Variability measurements}

Lipid variability was defined as intra-individual variability in lipid values between visits and only lipid profile values at least three months after discharge were included in this study to avoid the acute fluctuation in the peri-event period or due to initiation of statins. Various measurements of variability were performed: standard deviation (SD), coefficient of variation ( $\mathrm{CV}$, defined as $\mathrm{SD} /$ mean) and variability independent of the mean (VIM). VIM was calculated as $100 \times \mathrm{SD} / \mathrm{mean}^{x}$, with $x$ is the regression coefficient based on natural logarithm of SD on natural logarithm of mean. Besides, only patients with at least three post-baseline lipid measurements were included in the final analyses.

\section{Outcome assessment}

Patients were followed up by clinic revisit, E-mail or telephone conducted by trained nurses or doctors, who were blinded to the information of patients. The composite clinical endpoint included the following MACEs: unstable angina pectoris (UAP), myocardial infarction (MI), ischemic stroke, revascularization, and cardiovascular death (Table S1). Coronary revascularization was defined as percutaneous coronary intervention (PCI) or coronary artery bypass grafting (CABG) after 90 days of admission. During the follow-up period, 6 patients were lost to followup. Finally, a total of $254 \mathrm{FH}$ patients were included in the current study (Figure S1).

\section{Statistical analysis}

Continuous variables were presented as mean $\pm \mathrm{SD}$ or median (Q1-Q3 quartiles) and categorical variables were shown as number (percentage). The differences of clinical characteristics among groups were assessed using Student's $t$-test, Mann-Whitney U-test, chi-square test, Fisher's test, and ANOVA analysis as appropriate. Correlations of lipid parameters were evaluated by Pearson correlation analyses. The Cox proportional hazards regression model was performed to estimate the association between lipid variability and MACEs with hazard ratios (HRs) and $95 \%$ confidence intervals (CIs). The proportional hazards assumption was examined by Schoenfeld residuals test. Three different models were used to calculate each 1-SD increase in lipid variability: model 1 was the unadjusted model; model 2 was adjusted for age, sex, BMI, hypertension, diabetes, smoking, previous CAD, HbA1c, hsCRP and medications; and model 3 was additionally adjusted for mean visit-to-visit lipid values. The KaplanMeier method was applied to describe the MACEs. Subgroup analyses were performed by the stratification of age, sex, BMI, and the presence of hypertension or smoking. Age- and sex-adjusted restricted cubic spline (RCS) was used to assess the relationship between lipid variability with MACEs. All statistical analyses were performed with Stata version 14.0 (Stata Corp, Texas, USA). A P value $<0.05$ was considered statistically significant.

\section{Results}

\section{Baseline characteristics}

The baseline demographic and clinical characteristics of patients with FH are presented in Table 1 and Table S2. The mean age of participants was $47.91 \pm 12.63$ years old, in which $63.8 \%$ were male. Patients enrolled in the present study were divided into two groups: event group $(\mathrm{n}=22)$ and non-event group $(\mathrm{n}=232)$. Compared to those without MACEs, patients in event group had significantly higher concentrations of $\mathrm{Lp}$ (a) [38.10 (27.58-51.90) vs. 27.80 (13.06-51.94), $\mathrm{P}=0.040]$ and hs-CRP $[2.17(1.36-5.90) v s$. 
Table 1 Baseline characteristics of study subjects according to MACEs

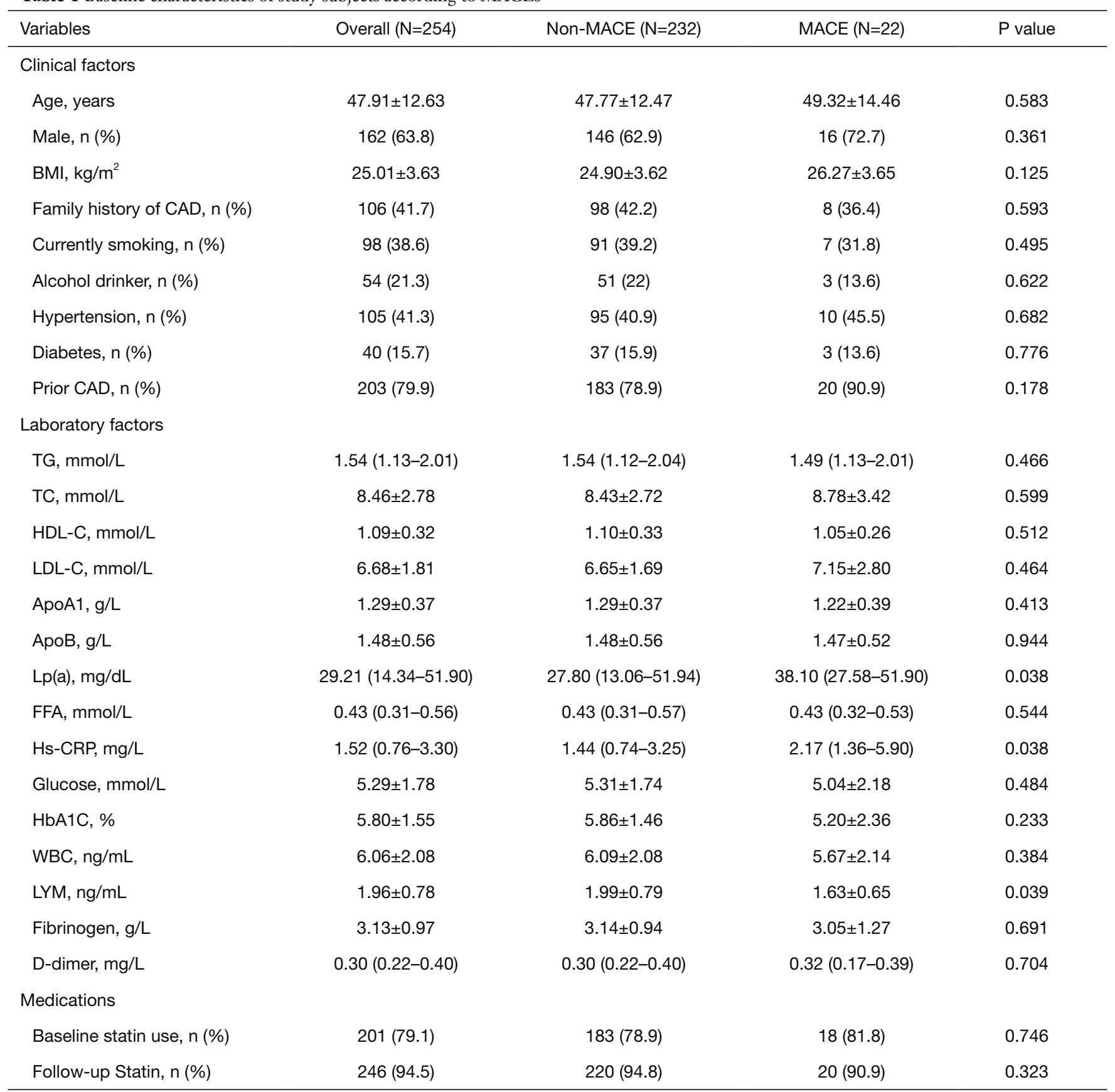

Data are presented as mean \pm SD, median (interquartile range) and number (\%). MACE, major adverse cardiac event; BMI, body mass index; CAD, coronary artery disease; TG, triglyceride; TC, total cholesterol; HDL-C, high-density lipoprotein cholesterol; LDL-C, lowdensity lipoprotein cholesterol; ApoA1, apolipoprotein A1; ApoB, apolipoprotein B; Lp(a), lipoprotein(a); hs-CRP, high sensitivity C-reactive protein; FFA: free fatty acid; HbA1c, glycosylated hemoglobin; WBC, white blood cell; LYM, lymphocyte.

1.44 (0.74-3.25), $\mathrm{P}=0.038]$. The mean concentration of LDL-C tended to be higher in the event group, but the difference was not significant. No difference was found with regard to the baseline TC, TC and HDL-C levels or the proportion of hypertension, diabetes and prior $\mathrm{CAD}$ between FH patients with and without MACEs. 
Table 2 Lipid profile variability measurements according to MACEs

\begin{tabular}{|c|c|c|c|}
\hline Variables & MACE $(\mathrm{N}=22)$ & Non-MACE (N=232) & $P$ value \\
\hline Mean follow-up levels (mmol/L) & $2.00 \pm 1.84$ & $1.67 \pm 1.05$ & 0.237 \\
\hline SD & $0.45 \pm 0.67$ & $0.35 \pm 0.41$ & 0.338 \\
\hline CV & $0.21 \pm 0.09$ & $0.20 \pm 0.16$ & 0.842 \\
\hline \multicolumn{4}{|l|}{ TC } \\
\hline Mean follow-up levels (mmol/L) & $6.18 \pm 3.26$ & $5.89 \pm 1.96$ & 0.560 \\
\hline SD & $1.26 \pm 0.88$ & $1.12 \pm 0.91$ & 0.542 \\
\hline CV & $0.22 \pm 0.14$ & $0.20 \pm 0.15$ & 0.434 \\
\hline \multicolumn{4}{|l|}{ HDL-C } \\
\hline Mean follow-up levels (mmol/L) & $0.91 \pm 0.19$ & $1.06 \pm 0.35$ & 0.064 \\
\hline SD & $0.16 \pm 0.09$ & $0.15 \pm 0.22$ & 0.919 \\
\hline CV & $0.18 \pm 0.12$ & $0.15 \pm 0.13$ & 0.244 \\
\hline VIM & $15.77 \pm 9.20$ & $14.76 \pm 19.74$ & 0.830 \\
\hline \multicolumn{4}{|l|}{ LDL-C } \\
\hline Mean follow-up levels (mmol/L) & $4.36 \pm 2.90$ & $4.16 \pm 1.71$ & 0.661 \\
\hline SD & $1.04 \pm 0.70$ & $1.03 \pm 0.78$ & 0.976 \\
\hline CV & $0.29 \pm 0.20$ & $0.26 \pm 0.18$ & 0.574 \\
\hline VIM & $74.81 \pm 66.66$ & $49.55 \pm 46.4$ & 0.036 \\
\hline
\end{tabular}

Data are presented as mean \pm SD. MACE, major adverse cardiac event; TG, triglyceride; TC, total cholesterol; HDL-C, high-density lipoprotein cholesterol; LDL-C, low-density lipoprotein cholesterol; Lp(a), lipoprotein(a); SD, standard deviation; CV, coefficient of variances; VIM, variability independent of mean.

\section{Visit-to-visit lipid variability}

Correlations between plasma visit-to-visit lipid variabilities are shown in Table S3. The SD of LDL-C was positively correlated with CV and VIM of LDL-C ( $\mathrm{r}=0.822$ and 0.916 , $\mathrm{P}<0.001$ for both). The SD of $\mathrm{Lp}(\mathrm{a})$ was also found to be correlated with CV and VIM of $\mathrm{Lp}(\mathrm{a})(\mathrm{r}=0.784$ and 0.832 , $\mathrm{P}<0.001$ for both). Part of variability parameters between LDL-C and $\mathrm{Lp}$ (a) were significantly but weekly correlated with each other. For instance, the VIM of Lp(a) was weekly correlated with the CV and VIM of LDL-C ( $\mathrm{r}=0.155$, $\mathrm{P}=0.014 ; \mathrm{r}=0.143, \mathrm{P}=0.023)$ but not with the SD of LDL-C $(\mathrm{r}=0.111, \mathrm{P}=0.078)$.

Table 2 shows the mean levels and variability parameters of lipids during follow-up. FH patients who developed MACEs had a non-statistical higher level of mean followup $\mathrm{Lp}(\mathrm{a})$. However, patients with MACEs had significantly higher SD of $\mathrm{Lp}$ (a) $(12.43 \pm 20.95$ vs. $7.32 \pm 9.94, \mathrm{P}=0.026)$. The CV and VIM of Lp(a) were also significantly higher in 

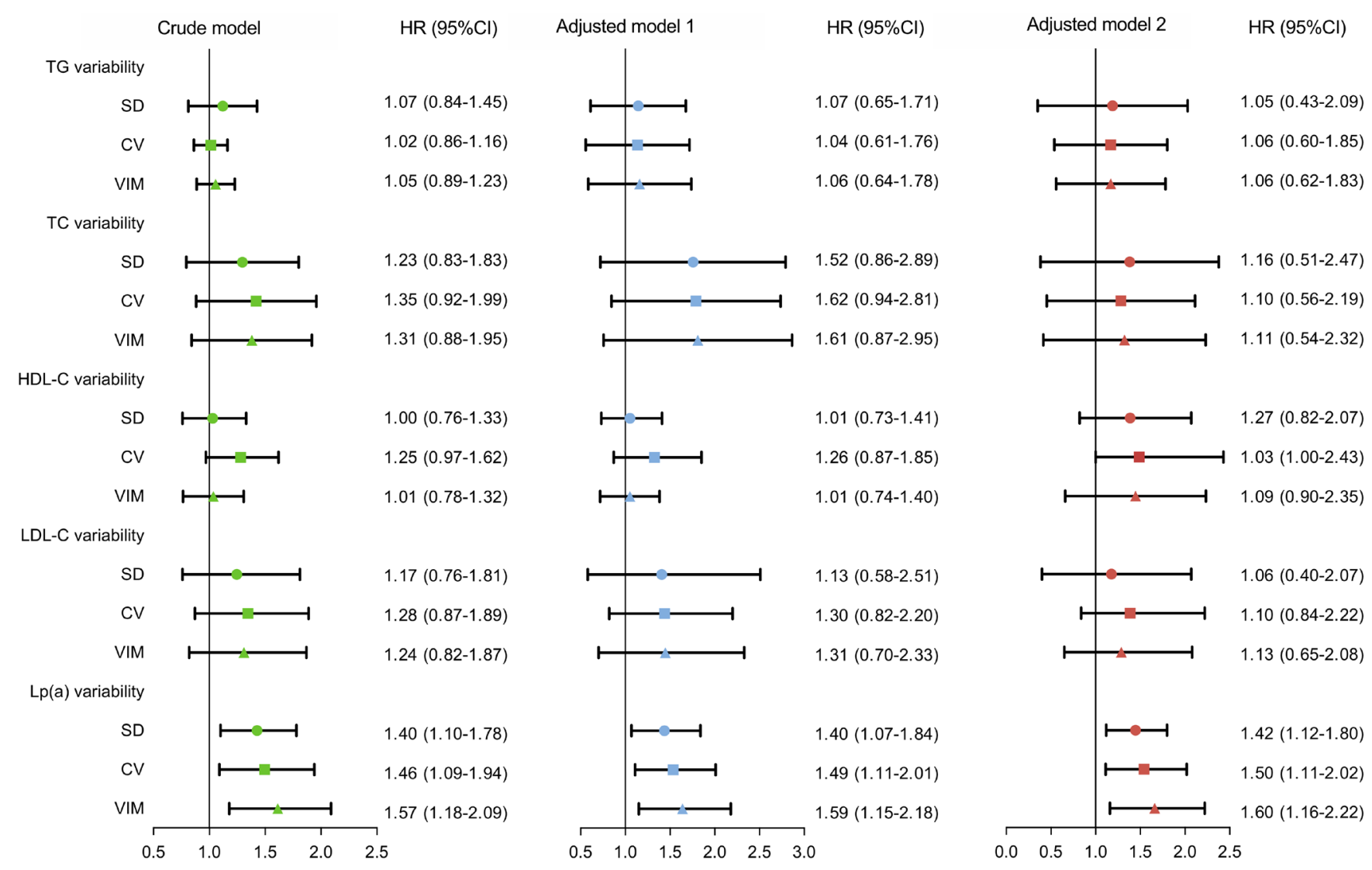

Figure 1 Risk of MACEs in unadjusted and adjusted Cox regression models for 1 standard deviation of lipid profile variability measurements. Hazard ratio of each 1 - standard deviation increase in variability. Model 1 adjusted for age, sex, body mass index, hypertension, diabetes, smoking, previous CAD, HbAlc, hs-CRP, and medications. Model 2 adjusted for model 1 plus mean TG, mean TC, mean LDL-C, mean HDL-C or mean Lp(a) level. MACE, major adverse cardiac event; TG, triglyceride; TC, total cholesterol; HDL-C, high-density lipoprotein cholesterol; LDL-C, low-density lipoprotein cholesterol; Lp(a), lipoprotein(a); CV, coefficient of variances; VIM, variability independent of mean.

patients with MACEs compared to those without MACEs. Regarding the variability parameters of TG, TC, HDL-C and LDL-C, no significant differences were observed between the two groups (all $\mathrm{P}>0.05$ ).

\section{Visit-to visit lipid variability and the risk of MACEs}

Over a mean follow-up period of 49.06 (25.34-68.48) months, 22 (8.7\%) events were recorded, representing 21.2 (13.8-32.1) events per 1,000 person-years. These events included 2 MI, 2 stroke, 10 coronary revascularization, 3 UAP and 5 cardiac death. As shown in Figure 1, univariate Cox proportional hazard regression analysis showed that every 1-SD increase in VIM of $\mathrm{Lp}(\mathrm{a})$ was positively associated with MACEs (HR: 1.57, 95\% CI: 1.18-2.09,
$\mathrm{P}=0.002)$. After adjustment for potential confounding factors, a 1-SD increment in VIM of $\mathrm{Lp}$ (a) increased the risk of MACEs by 59\% (HR: 1.59, 95\% CI: 1.15-2.18, $\mathrm{P}=0.005)$. The association was persisted after additional adjustment for mean follow-up Lp(a) levels (HR: 1.60; 95\% CI: 1.16-2.22; $\mathrm{P}=0.005)$. Similar relationships were observed between $\mathrm{SD}$ and $\mathrm{CV}$ of $\mathrm{Lp}(\mathrm{a})$ and the risk of MACEs. The Schoenfeld residual test revealed that the proportional hazards assumption was not violated with respect to $\mathrm{SD}$ of $\mathrm{Lp}(\mathrm{a})(\mathrm{P}=0.980), \mathrm{CV}$ of $\mathrm{Lp}(\mathrm{a})(\mathrm{P}=0.693)$, and VIM of $\mathrm{Lp}(\mathrm{a})(\mathrm{P}=0.833)$. There was a trend towards a higher risk of MACEs by variability in TG, TC, HDL-C and LDL-C, but these did not reach statistical significance in both univariate and multivariate Cox regression analysis (all $\mathrm{P}>0.05$ ). 
The Kaplan-Meier curves for the occurrence of MACEs between different $\mathrm{Lp}(\mathrm{a})$ variability levels are presented in Figure 2. The patients in tertiles 3 of Lp(a)-SD [high Lp(a) variability] had a worse event-free survival than those in tertile 1 [low $\mathrm{Lp}(\mathrm{a})$ variability]. The highest tertiles in $\mathrm{CV}$ and VIM of $\mathrm{Lp}(\mathrm{a})$ also showed a higher risk for MACEs compared to the lowest tertiles. RCS showed an increasing nonlinear trend in $\mathrm{Lp}(\mathrm{a})$ variability parameters with MACEs.

We also performed subgroup analyses stratified by baseline demographics and clinical characteristics (Tables S4-S7). The associations of Lp(a) variability parameters and MACEs were consistent across subgroups defined by age, sex, BMI, hypertension, and diabetes (all $\mathrm{P}<0.05)$. The trend for associations between VIM of LDL-C and MACEs were only observed in patients older than 50 years $(\mathrm{P}<0.05)$, without obesity and never smoking (all $\mathrm{P}<0.05)$. Besides, the SD of LDL-C was positively associated with MACEs in patients without obesity and never smoking (all $\mathrm{P}<0.05)$.

\section{Discussion}

The clinical characteristics were quite heterogeneous among patients with $\mathrm{FH}$, making it a pivotal issue to explore the potential prognostic predicator for further prevention and treatment (20). A growing body of evidence showed that intra-individual variability in biological indicators may be of importance to cardiovascular risk assessment $(16,17)$. Considering the need of extending the lipoprotein profile variability and no study has assessed the prognostic role of visit-to-visit lipid variability in patients with $\mathrm{FH}$, we investigated the association between lipid variability and MACEs in FH patients with standard lipidlowering therapy. The results indicated that visit-to-visit $\mathrm{Lp}(\mathrm{a})$ variability was a powerful and independent predictor of cardiovascular outcomes and each 1-SD increment in $\mathrm{Lp}$ (a) variability increased the risk of MACEs by $42 \%$, $50 \%$, and $60 \%$ when measured by SD, CV, and VIM, respectively. These associations remained significant even after adjustment for possible confounding factors including the mean follow-up $\mathrm{Lp}$ (a) level, and was consistent across all subgroups. of interest, the variability of LDL-C was not associated with a higher risk of cardiovascular events. To the best of our knowledge, in patients with $\mathrm{FH}$, this is the first study of the association between visit-to-visit lipid variability and MACEs and this finding added the novel information of the prognostic value of lipid variability and given new insight into serial monitoring of lipid in patients with FH.

Recently, variability in some biological parameters has been recognized as novel independent risk factors for cardiovascular outcomes $(21,22)$. Visit-to-visit BP variability is one of the mostly studied biological markers that each 5 - $\mathrm{mmHg}$ increase in SD of systolic BP increased the risk of cardiac mortality by $22 \%$ (21). As with BP systems, serum lipid levels also reflect a dynamic and homeostatic balance of synthesis and lipid clearance (23). Kim et al. (16) enrolled three million Korean general population and showed that the higher TC variability independently predicted adverse health-related outcomes. In a post-hoc analysis of the TNT trial including 9,572 patients with known CAD, each SD increase of visit-to-visit LDL-C was associated with $10-20 \%$ increased risk for MACEs after adjusted for trial medications and achieved LDL-C levels (17). Another study demonstrated that both elevated LDL-C and HDL-C variability were associated with the incidence of 5 -year MACEs in 130 patients with ST-segment elevation MI (24). However, the relationship between variability in $L p(a)$ and the risk of MACEs has yet to be investigated.

$\mathrm{FH}$ patients are characterized by lifetime elevated plasma LDL-C levels and higher risk for cardiovascular morbidity and mortality, highlighting the clinical importance of preventive strategies in this population (8). Although several baseline biochemical indexes were investigated as determinants of MACEs, there was no study evaluating the prognostic role of lipid variability in patients with $\mathrm{FH}$. In the present study, we found that all $\mathrm{Lp}$ (a) variability measurements (SD, CV, and VIM) were associated with increased risk for MACEs after adjusting for the possible confounding factors. This might be an interesting finding since emerging data have shown that the high inherit elevated plasma $\mathrm{Lp}(\mathrm{a})$ concentration was found in the one third of $\mathrm{FH}$ patients and was considered as a possible cause of clinical FH $(10,12,25)$. Considering that neither statin nor ezetimibe lowers $\mathrm{Lp}(\mathrm{a})$, FH patients with high $\mathrm{Lp}$ (a) levels remain at increased residual risk (10). Our study may extend this notion that visit-to-visit $\mathrm{Lp}(\mathrm{a})$ variability was also a prognostic factor for MACEs, suggesting that all individuals with $\mathrm{FH}$ should have their $\mathrm{Lp}$ (a) measured regularly in order to identify those with higher risk of adverse events.

Another interesting result of the present study was that variability in LDL-C showed a positive but non-statistically significant association with MACEs. In subgroup analysis, LDL-C variability could predict future MACEs in older FH patients or patients without obesity or smoking. The limited 

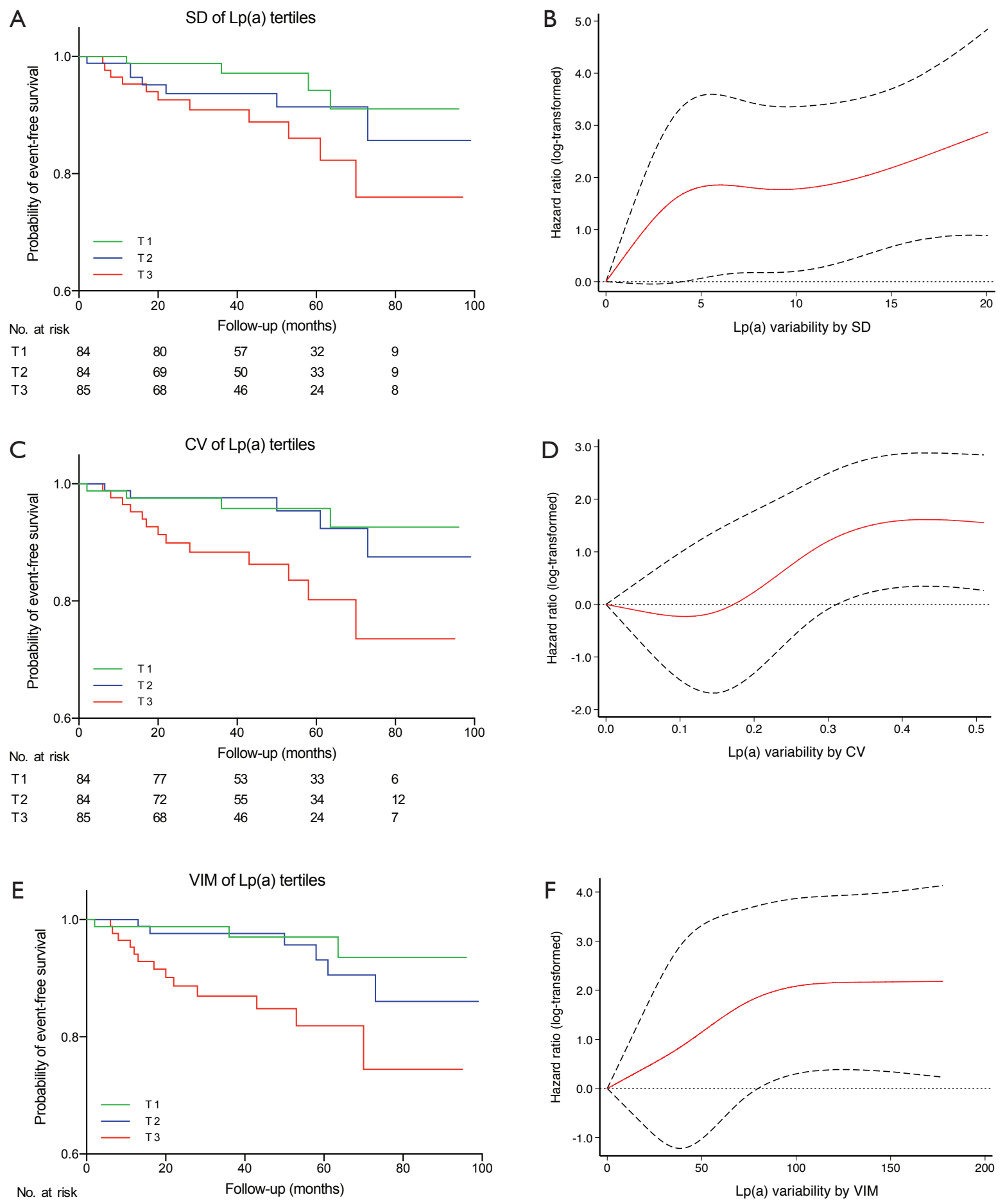

$\begin{array}{llllll}\text { T1 } & 84 & 77 & 53 & 30 & 5 \\ \text { T2 } & 84 & 75 & 59 & 36 & 16 \\ \text { T3 } & 85 & 65 & 43 & 22 & 5\end{array}$

Figure 2 Kaplan-Meier cumulative incidence of MACEs according to Lp(a) variability (A,C,E). Adjusted restricted cubic spline plot of the hazard ratio of MACEs against $\mathrm{Lp}(\mathrm{a})$ variability (B,D,F). Lp(a), lipoprotein(a); MACE, major adverse cardiovascular events. The solid line represents hazard ratio and the dashed lines represent the $95 \%$ confidence intervals. SD, standard deviation; CV, coefficient of variances; VIM, variability independent of mean. 
statistical power might attribute to the limited sample size and the heterogeneous characteristics of $\mathrm{FH}$ patients (8). Another possible reason was that the treatment adherence of $\mathrm{FH}$ patients was relatively higher than previous studies which might lead to less LDL-C variability $(17,18)$. Besides, the LDL-C levels were significantly higher in FH patients than general population in previous studies $(17,18)$. Anyway, the finding of the present study showed that monitoring $\mathrm{Lp}$ (a) might be more effective to stratify cardiovascular risk in FH patients than LDL-C levels alone during followup period. Nevertheless, studies about the association of LDL-C variability and clinical outcomes are of great importance to understand the mechanistic links between lipids and CAD, and thus lead to more efficacious therapy. For FH patients who receive PCSK9 inhibitors, evaluating lipid variability is a vital issue because some studies reported that LDL-C variability of every 4-week dosing was higher compared with every 2-week dosing PCSK9 inhibitors (26). More studies are warranted to clarify the prognostic role of LDL-C variability in patients with $\mathrm{FH}$.

The exact mechanism responsible for the association between lipid variability, especially $L p(a)$, and MACEs has not been fully clarified. However, several hypotheses have been proposed. First, plasma LDL particles could penetrate the endothelium of arterial walls and cause inflammation as well as endothelial injury (27). Previous studies have showed that higher lipid variability was positively associated with endothelial dysfunction (28). Second, the increase in the lipid variability might induce a repeated process of dissolution and crystallization of the cholesterol within coronary plaques $(29,30)$. The fluctuations in the composition of atherosclerotic plaques could impair the plaque stability, thereby leading to plaque rupture and causing cardiovascular events (18). Another possibility is that higher lipid variability may be an epiphenomenon linking to other systemic conditions which could worsen clinical outcome, such as body weight and diabetes (13). It was reported that a higher LDL-C variability was associated with higher fluctuation of body weight that could increase cardiovascular or mortality risk $(17,22)$. Furthermore, genetic factors related to lipid metabolism may also contribute to lipid variability. Recent studies have reported that apolipoprotein $\mathrm{E}$ genetic polymorphisms may account for the variability in LDL-C $(31,32)$. Finally, considering the close association between plasma lipid concentration and lipid-lowering agents, some studies suggested that poor adherence to medications could link lipid variability with increased risk of clinical outcomes $(13,18)$. However, in our study most patients still used statins during follow-up. Besides, in accordance with previous studies, the association between variability and MACEs remained significant even after adjusting for mean lipid levels and statin use $(16,17)$. Thus, based on the emerging notion that fluctuating lipid may be more insidious than stable cholesterol, regular monitoring could be of great importance in clinical practice for patients with $\mathrm{FH}$ (13).

Although this was the first study assessing the relation between visit-to-visit variability of lipid and MACEs in FH patients, several limitations also should be acknowledged. The major limitation is that sample size of this study is relatively small. Therefore, we could not perform subgroup analyses for each MACE event. Secondly, the follow-up lipid measurement schedule was not standardized and based on the discretion of physicians as most previous studies $(23,24)$. Moreover, the total numbers of measurement were different between patients. To minimize the effect of this limitation, we only included patients with more than three lipid measurements. Lastly, many medications could affect lipids variability. For example, intermittent use of steroids which can increase cholesterol without increasing MACE risk. Unfortunately, we did not have information of using other medication except lipid-lowering drugs. Additional larger and longitudinal studies with more measurements are warranted to confirm our findings.

\section{Conclusions}

In conclusion, we showed for the first time that in $\mathrm{FH}$ patients receiving standard lipid-lowering therapy, higher visit-to-visit $L p(a)$ variability was significantly and independently associated with increased risk for MACEs. Thus, the current study added novel information regarding the prognostic importance of lipid variability as a CAD risk factor. Future studies are needed to corroborate these findings and to identify the mechanisms promoting lipoprotein variability, including its therapeutic implications.

\section{Acknowledgments}

Funding: This work was supported by the Capital Health Development Fund (201614035), CAMS Major Collaborative Innovation Project (2016-I2M-1-011) and Fundamental Research Funds for the Central Universities (3332019135), the Fundamental Research Funds for the Central Universities (2019-XHQN09) and the Youth Research Fund of Peking Union Medical College (2019-F11). 


\section{Footnote}

Reporting Checklist: The authors have completed the STROBE reporting checklist. Available at http://dx.doi. org/10.21037/atm-20-5324

Data Sharing Statement: Available at http://dx.doi. org/10.21037/atm-20-5324

Conflicts of Interest: All authors have completed the ICMJE uniform disclosure form (available at http://dx.doi. org/10.21037/atm-20-5324). The authors have no conflicts of interest to declare.

Ethical Statement: The authors are accountable for all aspects of the work in ensuring that questions related to the accuracy or integrity of any part of the work are appropriately investigated and resolved. The study was conducted in accordance with the Declaration of Helsinki (as revised in 2013). The ethical approval of this study was obtained from the ethical review board of the Fuwai Hospital \& National Center for Cardiovascular Diseases, Beijing, China (approval ID: 2013-442). All patients enrolled in the study provided written informed consent.

Open Access Statement: This is an Open Access article distributed in accordance with the Creative Commons Attribution-NonCommercial-NoDerivs 4.0 International License (CC BY-NC-ND 4.0), which permits the noncommercial replication and distribution of the article with the strict proviso that no changes or edits are made and the original work is properly cited (including links to both the formal publication through the relevant DOI and the license). See: https://creativecommons.org/licenses/by-nc-nd/4.0/.

\section{References}

1. Mach F, Baigent C, Catapano AL, et al. 2019 ESC/EAS Guidelines for the management of dyslipidaemias: lipid modification to reduce cardiovascular risk. Eur Heart J 2020;41:111-88.

2. Arnett DK, Blumenthal RS, Albert MA, et al. 2019 ACC/AHA Guideline on the Primary Prevention of Cardiovascular Disease: Executive Summary: A Report of the American College of Cardiology/American Heart Association Task Force on Clinical Practice Guidelines. J Am Coll Cardiol 2019;74:1376-414.

3. Ference BA, Ginsberg HN, Graham I, et al. Low-density lipoproteins cause atherosclerotic cardiovascular disease. 1. Evidence from genetic, epidemiologic, and clinical studies. A consensus statement from the European Atherosclerosis Society Consensus Panel. Eur Heart J 2017;38:2459-72.

4. Hsia J, MacFadyen JG, Monyak J, et al. Cardiovascular event reduction and adverse events among subjects attaining low-density lipoprotein cholesterol $<50 \mathrm{mg} / \mathrm{dl}$ with rosuvastatin. The JUPITER trial (Justification for the Use of Statins in Prevention: an Intervention Trial Evaluating Rosuvastatin). J Am Coll Cardiol 2011;57:1666-75.

5. Liu DJ, Peloso GM, Yu H, et al. Exome-wide association study of plasma lipids in $>300,000$ individuals. Nat Genet 2017;49:1758-66.

6. Jin JL, Cao YX, Zhang HW, et al. Lipoprotein(a) and Cardiovascular Outcomes in Patients With Coronary Artery Disease and Prediabetes or Diabetes. Diabetes Care 2019;42:1312-8.

7. Li JJ, Li S, Zhu CG, et al. Familial Hypercholesterolemia Phenotype in Chinese Patients Undergoing Coronary Angiography. Arterioscler Thromb Vasc Biol 2017;37:570-9.

8. Nordestgaard BG, Chapman MJ, Humphries SE, et al. Familial hypercholesterolaemia is underdiagnosed and undertreated in the general population: guidance for clinicians to prevent coronary heart disease: consensus statement of the European Atherosclerosis Society. Eur Heart J 2013;34:3478-90a.

9. Li S, Wu NQ, Zhu CG, et al. Significance of lipoprotein(a) levels in familial hypercholesterolemia and coronary artery disease. Atherosclerosis 2017;260:67-74.

10. Langsted A, Kamstrup PR, Benn M, et al. High lipoprotein(a) as a possible cause of clinical familial hypercholesterolaemia: a prospective cohort study. Lancet Diabetes Endocrinol 2016;4:577-87.

11. Seed M, Hoppichler F, Reaveley D, et al. Relation of serum lipoprotein(a) concentration and apolipoprotein(a) phenotype to coronary heart disease in patients with familial hypercholesterolemia. N Engl J Med 1990;322:1494-9.

12. Cao YX, Jin JL, Guo YL, et al. Baseline and on-statin treatment lipoprotein(a) levels for predicting cardiovascular events in patients with familial hypercholesterolemia. Atherosclerosis 2019;291:27-33.

13. Simpson WG. Biomarker variability and cardiovascular disease residual risk. Curr Opin Cardiol 2019;34:413-7.

14. Gosmanova EO, Mikkelsen MK, Molnar MZ, et al. Association of Systolic Blood Pressure Variability With Mortality, Coronary Heart Disease, Stroke, and Renal 
Disease. J Am Coll Cardiol 2016;68:1375-86.

15. Tsuji H, Larson MG, Venditti FJ, Jr., et al. Impact of reduced heart rate variability on risk for cardiac events. The Framingham Heart Study. Circulation 1996;94:2850-5.

16. Kim MK, Han K, Kim HS, et al. Cholesterol variability and the risk of mortality, myocardial infarction, and stroke: a nationwide population-based study. Eur Heart J 2017;38:3560-6.

17. Bangalore S, Breazna A, DeMicco DA, et al. Visit-to-visit low-density lipoprotein cholesterol variability and risk of cardiovascular outcomes: insights from the TNT trial. J Am Coll Cardiol 2015;65:1539-48.

18. Clark D 3rd, Nicholls SJ, St John J, et al. Visit-tovisit cholesterol variability correlates with coronary atheroma progression and clinical outcomes. Eur Heart J 2018;39:2551-8.

19. Besseling J, Kindt I, Hof M, et al. Severe heterozygous familial hypercholesterolemia and risk for cardiovascular disease: a study of a cohort of 14,000 mutation carriers. Atherosclerosis 2014;233:219-23.

20. Miname MH, Bittencourt MS, Moraes SR, et al. Coronary Artery Calcium and Cardiovascular Events in Patients With Familial Hypercholesterolemia Receiving Standard Lipid-Lowering Therapy. JACC Cardiovasc Imaging 2019;12:1797-804.

21. Wang J, Shi X, Ma C, et al. Visit-to-visit blood pressure variability is a risk factor for all-cause mortality and cardiovascular disease: a systematic review and metaanalysis. J Hypertens 2017;35:10-7.

22. Bangalore S, Fayyad R, Laskey R, et al. Body-Weight Fluctuations and Outcomes in Coronary Disease. N Engl J Med 2017;376:1332-40.

23. Baber U, Halperin JL. Variability in low-density lipoprotein cholesterol and cardiovascular risk: should consistency be a new target? J Am Coll Cardiol 2015;65:1549-51.

Cite this article as: Cao $\mathrm{YX}, \mathrm{Li} \mathrm{L}$, Zhang HW, Jin JL, Liu HH, Guo YL, Wu NQ, Zhu CG, Dong Q, Xu RX, Sun J, Li JJ. Visit-to-visit variability of lipid and cardiovascular events in patients with familial hypercholesterolemia. Ann Transl Med 2021;9(7):556. doi: 10.21037/atm-20-5324
24. Boey E, Gay GM, Poh KK, et al. Visit-to-visit variability in LDL- and HDL-cholesterol is associated with adverse events after ST-segment elevation myocardial infarction: A 5-year follow-up study. Atherosclerosis 2016;244:86-92.

25. Sun D, Cao YX, Li S, et al. A modified algorithm with lipoprotein(a) added for diagnosis of familial hypercholesterolemia. Clin Cardiol 2019;42:988-94.

26. Giugliano RP, Desai NR, Kohli P, et al. Efficacy, safety, and tolerability of a monoclonal antibody to proprotein convertase subtilisin/kexin type 9 in combination with a statin in patients with hypercholesterolaemia (LAPLACETIMI 57): a randomised, placebo-controlled, doseranging, phase 2 study. Lancet 2012;380:2007-17.

27. Gutierrez E, Flammer AJ, Lerman LO, et al. Endothelial dysfunction over the course of coronary artery disease. Eur Heart J 2013;34:3175-81.

28. Smit RA, Trompet S, Sabayan B, et al. Higher Visit-toVisit Low-Density Lipoprotein Cholesterol Variability Is Associated With Lower Cognitive Performance, Lower Cerebral Blood Flow, and Greater White Matter Hyperintensity Load in Older Subjects. Circulation 2016;134:212-21.

29. Abela GS, Vedre A, Janoudi A, et al. Effect of statins on cholesterol crystallization and atherosclerotic plaque stabilization. Am J Cardiol 2011;107:1710-7.

30. Phillips MC. Molecular mechanisms of cellular cholesterol efflux. J Biol Chem 2014;289:24020-9.

31. Lussier-Cacan S, Xhignesse M, Kessling AM, et al. Sources of variation in plasma lipid and lipoprotein traits in a sample selected for health. Am J Epidemiol 1999;150:1229-37.

32. Heath KE, Gudnason V, Humphries SE, et al. The type of mutation in the low density lipoprotein receptor gene influences the cholesterol-lowering response of the HMG-CoA reductase inhibitor simvastatin in patients with heterozygous familial hypercholesterolaemia. Atherosclerosis 1999;143:41-54. 


\section{Supplementary}

Table S1 International classification of diseases codes for MACE

$\begin{array}{ll}\begin{array}{l}\text { Diagnosis/procedure } \\ \text { Myocardial infarction }\end{array} & \begin{array}{l}\text { ICD-9-CM codes/ICD-10 } \\ \text { codes } \\ \text { I21 } \\ \text { Angina pectoris }\end{array} \\ \text { Cardiovascular death } & \begin{array}{l}\text { I20 } \\ \text { I20-I51 due to I00-I10, I11, I13, }\end{array} \\ \text { Ischemic stroke } & \text { I63, I64 } \\ \text { Percutaneous coronary } & \text { ICD-9-CM 36.06/36.07 } \\ \text { intervention } & \\ \text { Coronary artery bypass grafting } & \text { ICD-9-CM 36.1-36.19 }\end{array}$

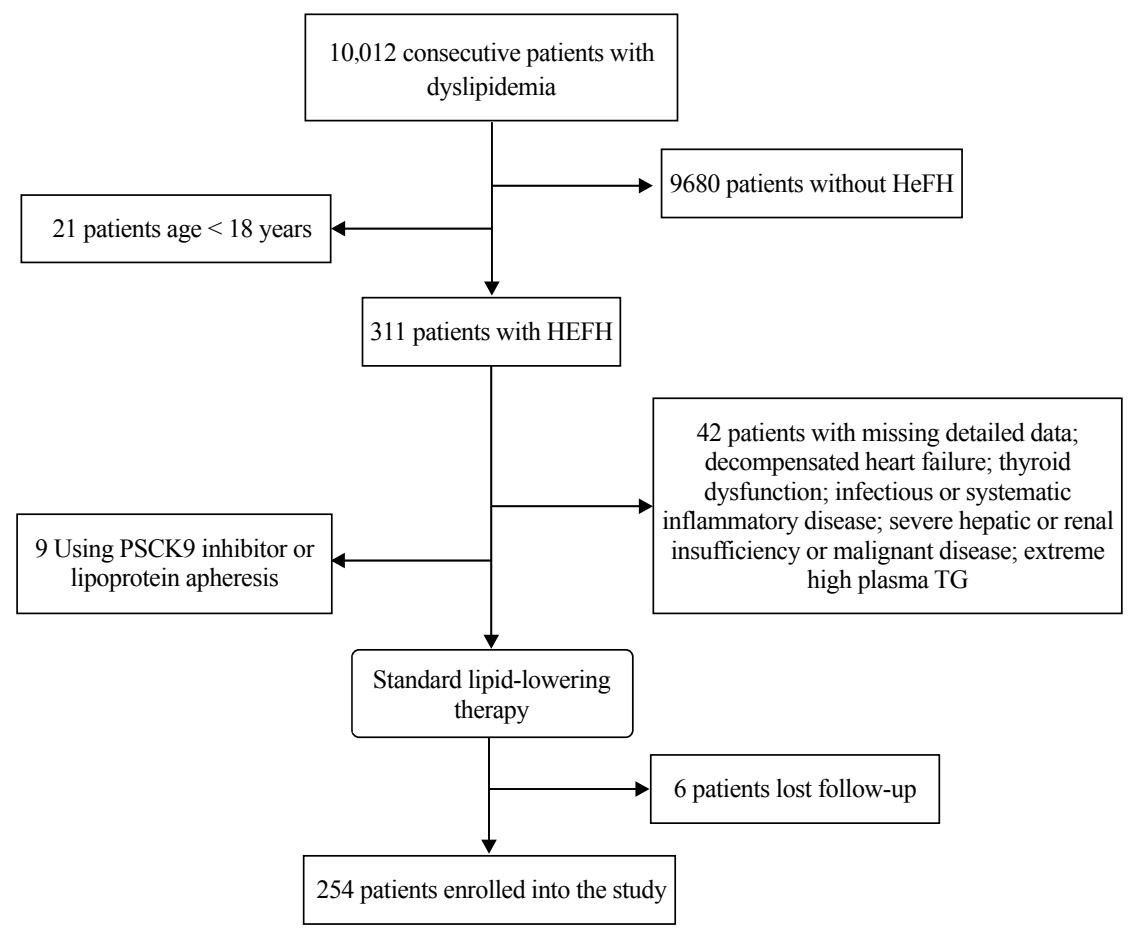

Figure S1 The flowchart of this study. 
Table S2 Correlations between lipid variability parameters

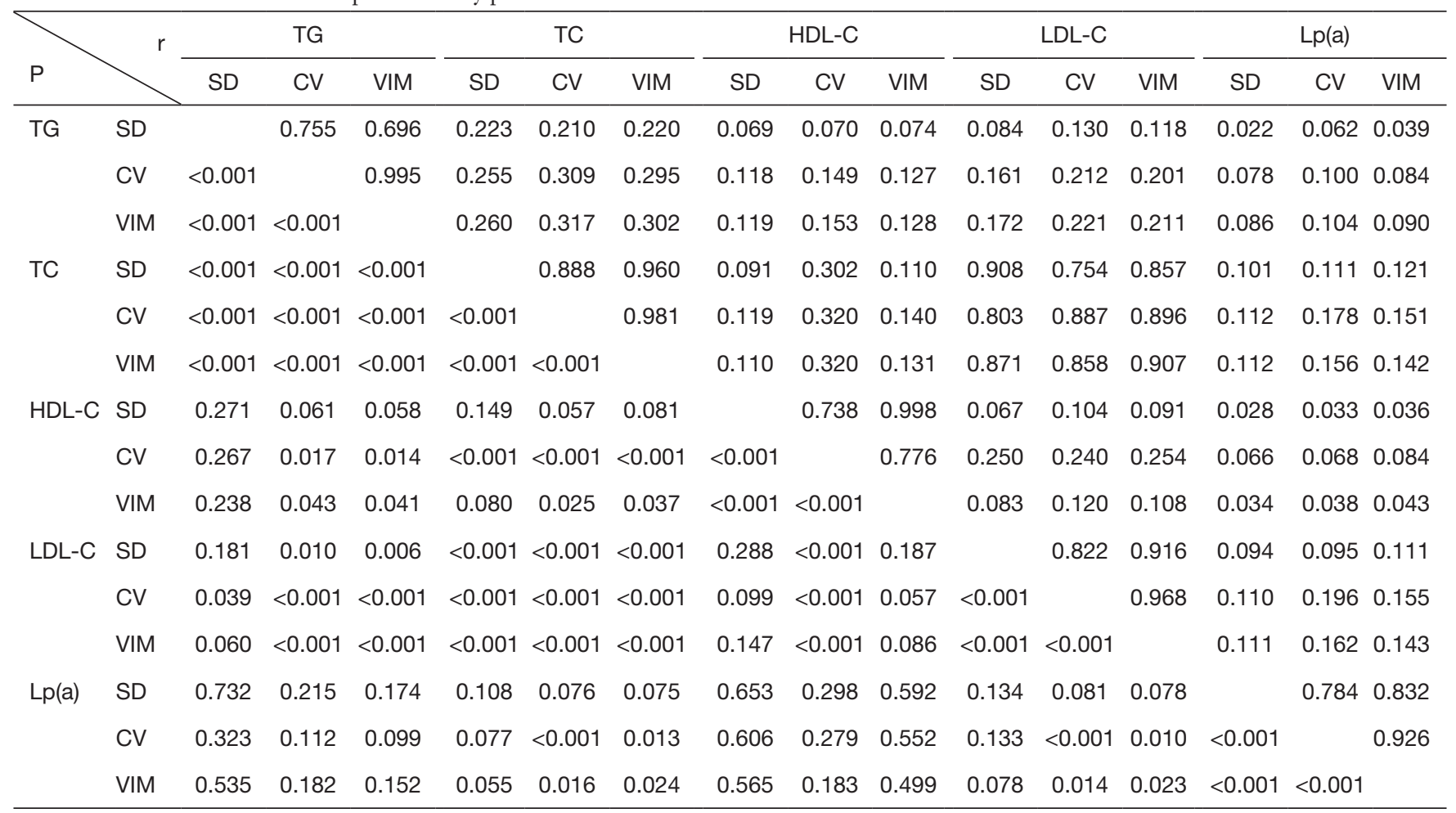

Table S3 Hazard ratio of TG variability for MACE by subgroup

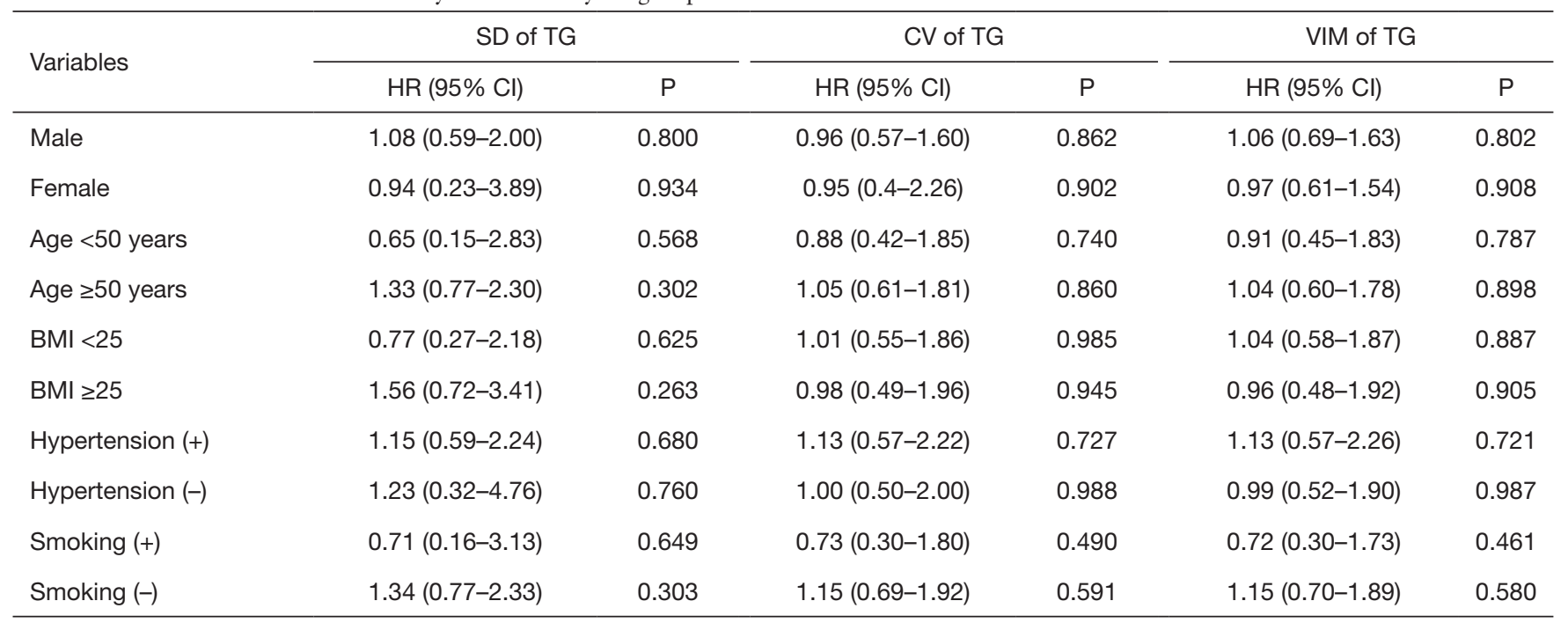


Table S4 Hazard ratio of TC variability for MACE by subgroup

\begin{tabular}{|c|c|c|c|c|c|c|}
\hline Variables & \multicolumn{2}{|l|}{ SD of TC } & \multicolumn{2}{|c|}{ CV of TC } & \multicolumn{2}{|c|}{ VIM of TC } \\
\hline Male & $1.18(0.74-1.89)$ & 0.479 & $1.25(0.79-1.97)$ & 0.345 & $1.22(0.77-1.92)$ & 0.401 \\
\hline Female & $2.35(0.94-5.86)$ & 0.066 & $2.28(1.00-5.19)$ & 0.050 & $2.27(0.99-5.21)$ & 0.053 \\
\hline Age $<50$ years & 1.07 (0.82-1.39) & 0.609 & $1.05(0.80-1.37)$ & 0.736 & $1.05(0.81-1.36)$ & 0.729 \\
\hline $\mathrm{BMI}<25$ & $1.23(0.58-2.6)$ & 0.586 & $1.19(0.63-2.26)$ & 0.600 & $1.19(0.61-2.34)$ & 0.607 \\
\hline $\mathrm{BMI} \geq 25$ & $1.35(0.81-2.24)$ & 0.252 & $1.44(0.88-2.36)$ & 0.146 & $1.40(0.86-2.27)$ & 0.176 \\
\hline Hypertension (+) & $2.16(1.13-4.14)$ & 0.020 & $1.87(1.06-3.30)$ & 0.030 & $1.99(1.11-3.58)$ & 0.022 \\
\hline Hypertension (-) & 1.03 (0.97-1.09) & 0.372 & $1.12(0.58-2.19)$ & 0.734 & $1.03(0.53-2.01)$ & 0.935 \\
\hline
\end{tabular}

Table S5 Hazard ratio of HDL-C variability for MACE by subgroup

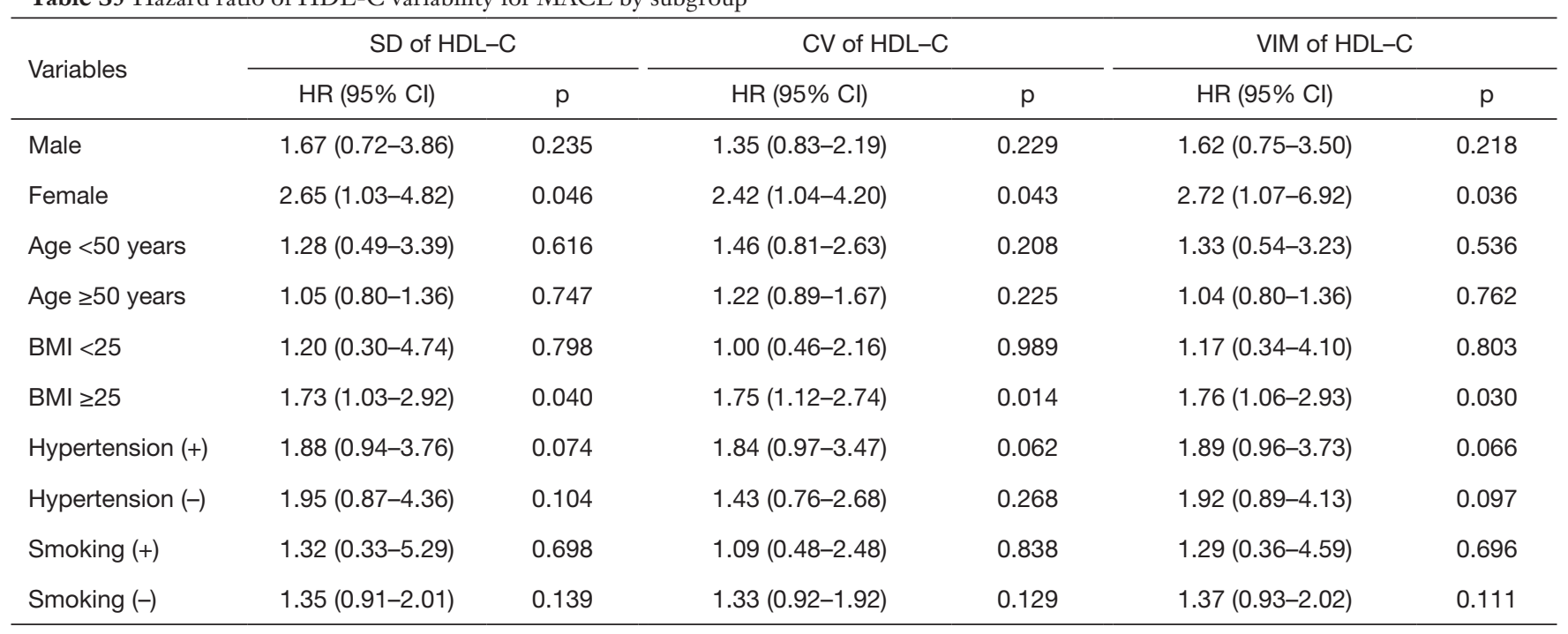


Table S6 Hazard ratio of LDL-C variability for MACE by subgroup

\begin{tabular}{|c|c|c|c|c|c|c|}
\hline Variables & \multicolumn{2}{|c|}{ SD of LDL-C } & \multicolumn{2}{|c|}{ CV of LDL-C } & \multicolumn{2}{|c|}{ VIM of LDL-C } \\
\hline Male & $1.03(0.61-1.75)$ & 0.911 & $1.20(0.74-1.96)$ & 0.454 & $1.12(0.69-1.82)$ & 0.654 \\
\hline Female & $1.98(0.78-5.02)$ & 0.152 & $1.85(0.83-4.11)$ & 0.133 & $1.90(0.84-4.32)$ & 0.126 \\
\hline Age $<50$ years & $1.05(0.77-1.42)$ & 0.764 & $1.01(0.60-1.70)$ & 0.978 & $1.01(0.81-1.26)$ & 0.951 \\
\hline BMI $<25$ & $1.21(0.56-2.58)$ & 0.631 & $1.40(1.04-1.90)$ & 0.029 & $1.37(1.02-1.85)$ & 0.037 \\
\hline $\mathrm{BMI} \geq 25$ & $1.39(0.76-2.56)$ & 0.290 & $1.39(0.82-2.34)$ & 0.218 & $1.39(0.81-2.38)$ & 0.237 \\
\hline Hypertension (+) & $1.55(0.85-2.84)$ & 0.152 & $1.48(0.88-2.51)$ & 0.140 & $1.51(0.89-2.56)$ & 0.130 \\
\hline Hypertension (-) & $0.95(0.48-1.91)$ & 0.890 & $1.17(0.60-2.28)$ & 0.657 & $1.03(0.53-2.00)$ & 0.921 \\
\hline
\end{tabular}

Table S7 Hazard ratio of $L p(a)$ variability for MACE by subgroup

\begin{tabular}{|c|c|c|c|c|c|c|}
\hline Variables & \multicolumn{2}{|c|}{ SD of Lp(a) } & \multicolumn{2}{|c|}{ CV of Lp(a) } & \multicolumn{2}{|c|}{ VIM of Lp(a) } \\
\hline Male & $1.35(1.02-1.78)$ & 0.038 & $1.48(1.06-2.07)$ & 0.020 & $1.51(1.12-2.05)$ & 0.007 \\
\hline Female & 1.29 (1.01-1.65) & 0.043 & $1.12(1.01-1.84)$ & 0.025 & 1.47 (1.03-2.36) & 0.044 \\
\hline Age $<50$ years & $1.29(1.04-2.05)$ & 0.036 & $1.22(1.02-1.98)$ & 0.042 & $1.40(1.05-2.33)$ & 0.037 \\
\hline $\mathrm{BMI}<25$ & $1.10(1.02-1.95)$ & 0.042 & $1.26(1.04-2.12)$ & 0.034 & $1.26(1.04-2.16)$ & 0.039 \\
\hline $\mathrm{BMI} \geq 25$ & 1.57 (1.12-2.20) & 0.009 & $1.69(1.05-2.72)$ & 0.030 & $1.90(1.25-2.87)$ & 0.002 \\
\hline Hypertension (+) & $1.18(0.42-3.32)$ & 0.750 & $1.16(1.04-1.98)$ & 0.032 & $1.19(1.05-2.18)$ & 0.038 \\
\hline Hypertension (-) & $1.36(0.98-1.88)$ & 0.069 & $1.82(1.15-2.89)$ & 0.011 & 1.65 (1.12-2.42) & 0.011 \\
\hline
\end{tabular}

